\title{
Energy non-linearity effects in the response of ionic crystal scintillators to X-rays with energy in the region of the K-absorptions edges: experimental results
}

\author{
L.F. Requicha Ferreira ${ }^{a}$, H.M.N.B.L. Ferreira ${ }^{a}$, J.F.C.A. Veloso ${ }^{a, b}$, \\ J.M.F. Dos Santos ${ }^{\mathrm{a}, *}$ \\ a Physics Department, University of Coimbra, Coimbra P-3004-516, Portugal \\ ${ }^{\mathrm{b}}$ Physics Department, University of Aveiro, Aveiro P-3810-193, Portugal
}

Received 24 March 2003; received in revised form 29 July 2003; accepted 30 August 2003

\begin{abstract}
The response of a YAP, $\mathrm{NaI}(\mathrm{Tl})$ and $\mathrm{BaF}_{2}$ scintillators to X-rays with energies around the $\mathrm{Y}$, I, and $\mathrm{Ba} \mathrm{K}$-absorption edges, respectively, was investigated. For all the scintillators, the amplitude response follows different linear trends for $\mathrm{X}$-ray energies below and above the respective K-edges, presenting a discontinuity at these energies. An abrupt decrease of about $3 \%, 5 \%$ and $2 \%$ were observed in the detector amplitude at the K-edges, for the YAP, the NaI(Tl) and the $\mathrm{BaF}_{2}$ scintillator, respectively, corresponding to a decrease of $0.5 \pm 0.1,1.7 \pm 0.3$ and $0.8 \pm 0.2 \mathrm{keV}$ in the energy calibration line. These discontinuities result in a region within $0.5 \pm 0.1,1.6 \pm 0.3$ and $0.9 \pm 0.2 \mathrm{keV}$ where the X-ray energy cannot be obtained unambiguously. The scintillation yields for X-rays present abrupt decreases of about $3 \%$, $4 \%$ and $2 \%$, respectively, at the K-edges. The measured non-linearity effects are significantly larger than those obtained for gaseous and semiconductor detectors. The higher amplitude non-linearity observed in NaI(Tl) is attributed to the larger light yield non-linearity in the electron response of this crystal.
\end{abstract}

(C) 2003 Elsevier B.V. All rights reserved.

PACS: $07.85 . \mathrm{Nc} ; 29.30 . \mathrm{Kv} ; 29.40 . \mathrm{Mc} ; 32.80 . \mathrm{Hd} ; 32.80 . \mathrm{t} ; 85.60 . \mathrm{Jb}$

Keywords: Inorganic scintillators; $\mathrm{NaI}(\mathrm{Tl}) ; \mathrm{BaF}_{2}$; YAP; Energy non-linearity; K-edge

\section{Introduction}

Non-linearities in the response of gaseous and semiconductor detectors to X-rays have been extensively investigated by both experimental and

*Corresponding author. Tel.: + 351-239-410667; fax: + 351239-829158.

E-mail address: jmf@gian.fis.uc.pt (J.M.F.dos Santos).
Monte Carlo studies. While for Xe and Ar gas detectors significant departure from linearity, in particular the detector amplitude, have been observed at the gas absorption edges (see Ref. [1] and references therein), for semiconductor detectors non-linearity effects were found to be negligible or non-existent [2-5]. For example, at the $\mathrm{Xe} \mathrm{L}_{\mathrm{III}^{-}}$and $\mathrm{K}$-absorption edges, an abrupt decrease of about $1.5 \%$ and $0.5 \%$, respectively, 
was found in the detector amplitude for different types of xenon-gas detectors [1], corresponding to a decrease of about 90 and $160 \mathrm{eV}$ in the energy calibration line. For an argon proportional counter, this decrease of was found to be about $70 \mathrm{eV}$ at the Ar K-edge [6]. Apart from the regions around the absorption edges, the amplitude response of gaseous detectors to X-rays was found to be linear within the experimental accuracy.

Monte Carlo calculations [1,7] have shown that non-linearities result from differences in the efficiencies for converting absorbed radiation into ionisation, for different atomic sub-shells. The efficiency for converting absorbed X-ray energies into ionisation is lower when photon interactions take place in atomic sub-shells with higher binding energy. When a new photo-ionisation channel becomes energetically accessible, the subsequent de-excitation cascade of the photoionised atoms result in a larger number of electron vacancies in the outermost sub-shells. Additionally, the overall kinetic energy of the photoelectron and other electrons emitted by the photoionised atom decreases, producing a smaller number of primary electrons during the thermalization process $[1,8]$. At still higher energies, the energy dissipated in establishing the cascade vacancies and the kinetic energy of electrons emitted by the photoionised atom is a smaller fraction of the total energy transferred to those electrons, and approximate energy linearity is restored. However, for semiconductor detectors the electrons of the outermost sub-shells are shared in covalent orbitals, and collective effects of the crystalline medium dominate the energy absorption processes, minimising any non-linearity $[2,3]$.

Non-linearities in the response of inorganic scintillators to X-rays have also been investigated, being $\mathrm{NaI}(\mathrm{Tl})$ the most studied one (see Ref. [9] and the references therein). While Leutz et al. [10] conclude that there are no intrinsic non-linearity effects in $\mathrm{NaI}(\mathrm{Tl})$, Valentine et al. [11] provide arguments disregarding that conclusion. Wayne et al. [12] present a detailed study on the X-ray scintillation yield, i.e. the light output response divided by the X-ray energy, as a function of the $\mathrm{X}$-ray energy for $\mathrm{NaI}(\mathrm{Tl})$, in particular in the region around the I K-edge, using a synchrotron radiation source. The X-ray scintillation yield presents an abrupt variation at the I K-edge and a continuous variation below and above the edge. This behaviour is typical for this type of ionic scintillators (see Ref. [9] and the references therein) and is similar to that presented by $w$-values, i.e., the average energy to produce an electron-ion pair, in xenon gaseous detectors. Dorenbos et al. [9] present a very detailed review on the nonlinearity studies in scintillators and also present experimental results for modern scintillators, such as LSO, YAP, $\mathrm{BaF}_{2}$ and others.

In general, the non-linearity studies refer to the X-ray scintillation yield as a function of the $\mathrm{X}$-ray energy and do not refer its effect on the detector amplitude response $[9,11]$. However, as discussed in Ref. [4], although the continuous variation of $w$ with the X-ray energy cannot be neglected, its effect on the detector amplitude linearity is small, so that, apart from the abrupt discontinuities near the subshell-thresholds, the detector amplitude varies almost linearly with the $\mathrm{X}$-ray energy. The same behaviour is expected to be present in scintillators. In particular, although the scintillation response may present a nearly linear behaviour, a significant non-linearity is expected to be observed at the medium absorption edges. Such significant discontinuities in the amplitude response of scintillators have not yet been reported.

On the other hand, the results presented for some modern scintillators, like YAP and $\mathrm{BaF}_{2}$, are scarce, and do not include a significant number of data for X-ray energies around the respective K-edges $[9,13]$.

In the present work we experimentally investigate the response of $\mathrm{NaI}(\mathrm{Tl}), \mathrm{BaF}_{2}$ and YAP scintillators to $\mathrm{X}$-rays with energies around the $\mathrm{K}$-edges of $\mathrm{I}, \mathrm{Ba}$ and $\mathrm{Y}$, respectively. Detector amplitude and scintillation yield behaviour are studied as a function of the X-ray energy. Photon responses have been calculated from measured electron responses, using dedicated Monte Carlo Codes $[11,14,15]$. The detailed scintillation response to X-rays with energies around the K-edge can be used as input for, or in combination with, these simulations, in order to have better assessment of electron responses at low energies 
(typically $<10 \mathrm{keV}$ ) as well as its contribution to the calculated photon response (e.g. see Ref. [15]).

\section{Experimental set-up and method}

Fig. 1 depicts the typical configuration of the excitation/detection system used in this work. The X-ray energies required for this study were generated by exciting K-fluorescence lines in selected target elements. The fluorescent targets in the shape of disks of about $3 \mathrm{~cm}$ in diameter and $1-\mathrm{cm}$ thick were selected on the basis of their availability and of their $\mathrm{K}_{\alpha}$-energies (Table 1 ). The targets were positioned $45^{\circ}$ to the detector axis while the radioactive source (either ${ }^{241} \mathrm{Cm} \gamma$-source or a ${ }^{109} \mathrm{Cd}$ X-ray source) was positioned at $90^{\circ}$.

The scintillators were coupled to photomultiplier tubes (PMT), being the PMT pulses directed through a charge preamplifier and an amplifier $(1-\mu \mathrm{s}$ integration and differentiation time-constants, 2- $\mu$ s for the $\mathrm{BaF}_{2}$ case) to a 1024 multichannel analyser. Both YAP and $\mathrm{BaF}_{2}$ detectors were home-made detectors, while the $\mathrm{NaI}(\mathrm{Tl})$ detector is commercially available. The $\mathrm{NaI}(\mathrm{Tl})$

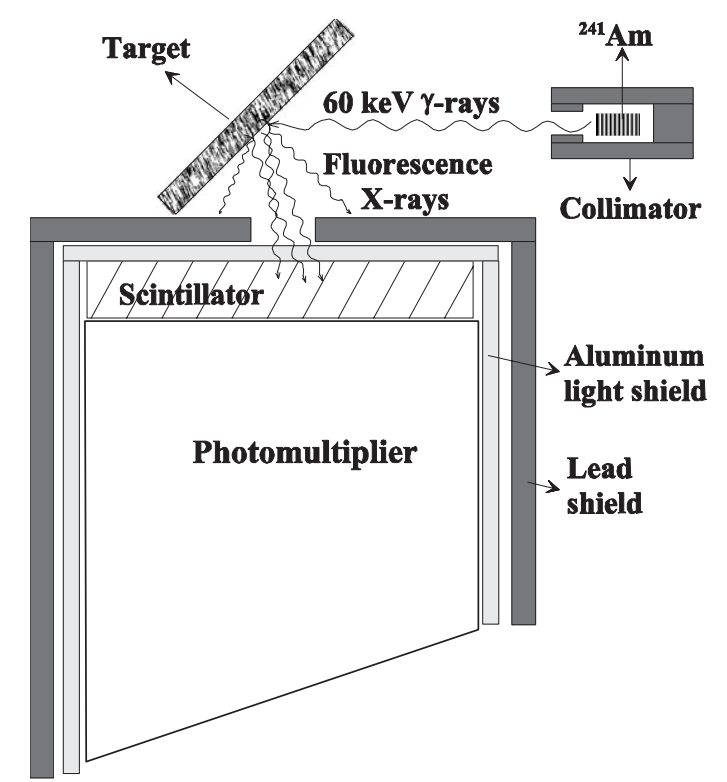

Fig. 1. Schematic of the detector and the source/target geometry.
Table 1

Characteristic radiation lines used in the experiment

\begin{tabular}{llll}
\hline K Lines & Ex $(\mathrm{keV})$ & K Lines & Ex $(\mathrm{keV})$ \\
\hline $\mathrm{Sr} \mathrm{K} \alpha$ & 14.142 & $\mathrm{Sb} \mathrm{K} \alpha$ & 26.274 \\
$\mathrm{Y} \mathrm{K} \alpha$ & 14.933 & $\mathrm{I} \mathrm{K} \alpha$ & 28.512 \\
$\mathrm{Zr} \mathrm{Ka}$ & 15.746 & $\mathrm{Cs} \mathrm{K} \alpha$ & 30.854 \\
$\mathrm{Nb} \mathrm{K} \alpha$ & 16.584 & $\mathrm{Ba} \mathrm{K} \alpha$ & 32.065 \\
$\mathrm{Mo} \mathrm{K} \alpha$ & 17.443 & $\mathrm{La} \mathrm{K} \alpha$ & 33.302 \\
$\mathrm{Ru} \mathrm{K} \alpha$ & 19.235 & $\mathrm{Ce} \mathrm{K} \alpha$ & 34.569 \\
$\mathrm{Rh} \mathrm{K} \alpha$ & 20.167 & $\mathrm{Pr} \mathrm{K} \alpha$ & 35.864 \\
$\mathrm{Pd} \mathrm{K} \alpha$ & 21.123 & $\mathrm{Nd} \mathrm{K} \alpha$ & 37.185 \\
$\mathrm{Ag} \mathrm{K} \alpha$ & 22.104 & $\mathrm{Sm} \mathrm{K} \alpha$ & 39.914 \\
$\mathrm{In} \mathrm{K} \alpha$ & 24.139 & $\mathrm{Gd} \mathrm{K} \alpha$ & 42.761 \\
$\mathrm{Sn} \mathrm{K} \alpha$ & 25.193 & $\mathrm{Dy} \mathrm{K} \alpha$ & 45.728 \\
& & $\mathrm{Er} \mathrm{K} \alpha$ & 48.818 \\
\hline
\end{tabular}

is an Harshaw Integral Line, with a $40-\mathrm{cm}^{2} \times$ $10-\mathrm{cm}$ crystal, the $\mathrm{BaF}_{2}$ detector consisted of a $20-\mathrm{cm}^{2} \times 5-\mathrm{cm}$ crystal coupled to a $5-\mathrm{cm}$ diameter PMT and the YAP detector consisted of a $13-\mathrm{cm}^{2} \times 2-\mathrm{mm}$ crystal, coupled to the PMT through optical grease.

The obtained pulse-height distributions were fitted to Gaussian functions superimposed on a linear background, using the least-squares fit method [16]. The peak-centroid and energy resolution were monitored as a function of the X-ray energy. By measuring at these discrete energies, the integral non-linearity in the detector is obtained, in opposition to the differential and integral non-linearities that could be measured using a continuously tuneable X-ray source, such as synchrotron radiation. However, this method has been used to measure the non-linearity in the response of Xe, Ar, Ge and CZT detectors around the L- and/or K-edges [1,4-6] and presented similar results to those obtained with other methods $[1,4]$. Additionally, as a single discontinuity may exist at the $\mathrm{K}$-edge, the analysis performed with this method is valid for the whole covered region.

Three different regions were considered, around $\mathrm{Y}$, I and $\mathrm{Ba} \mathrm{K}$-edges, respectively. A fixed X-ray line was monitored throughout each run, to check system stability and to evaluate the errors in the obtained peak-centroids. At least four independent runs were performed for each region to evaluate the overall error obtained in the discontinuity measurements. The count-rate in the detectors was 
maintained sufficiently low to make negligible any dead-time and pile-up effects, minimising any count rate-effects due to the abrupt increase in the absorption efficiency at the K-edges.

\section{Experimental results and discussion}

In Fig. 2 the $\mathrm{K}_{\alpha}$-peak centroids are depicted as a function of the energy for the regions around the Y, I and Ba K-absorption edges (17.037, 33.164 and $37.410 \mathrm{keV}$, respectively). The least-squares fit
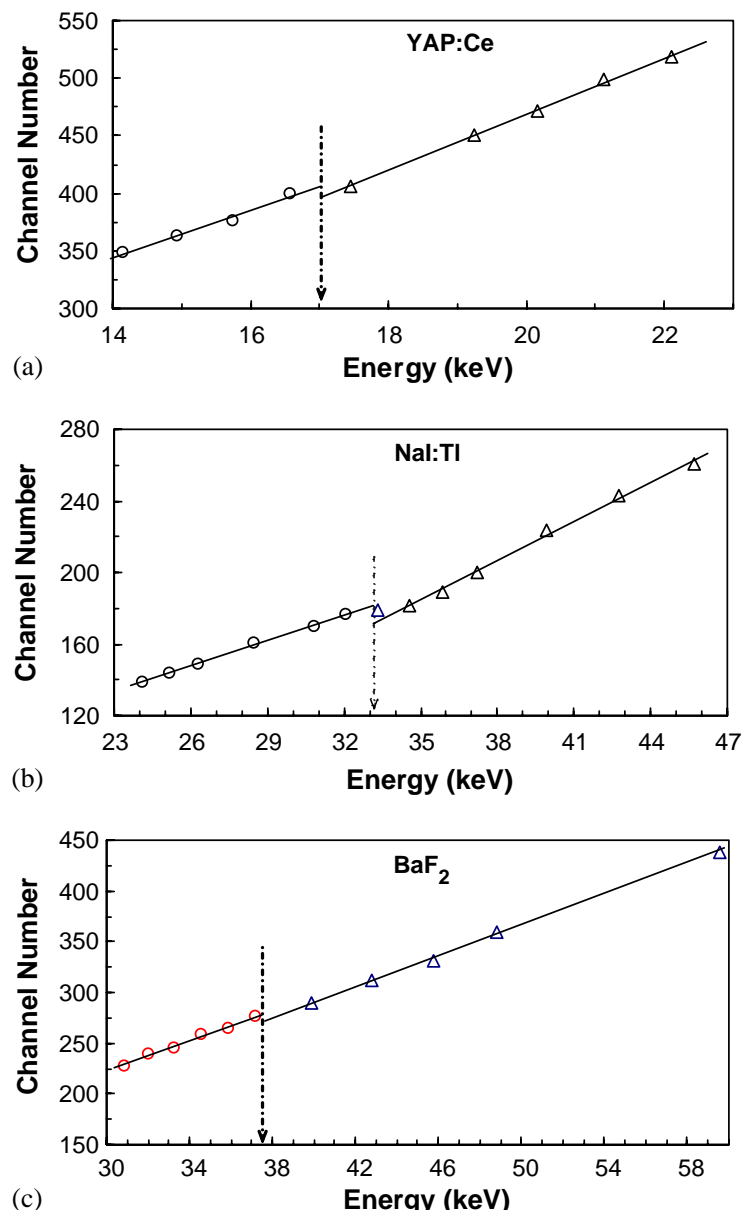

Fig. 2. Detector relative amplitude $(O, \Delta)$ as a function of the X-ray energy, $E_{x}$ : (a) for YAP, (b) $\mathrm{NaI}(\mathrm{Tl})$ and (c) $\mathrm{BaF}_{2}$ scintillators. The solid straight lines represent linear leastsquare fits to each set of data below $(O)$ and above $(\Delta)$ the respective $\mathrm{K}$-absorption edges. of straight lines to each set of data below and above the K-edges are also depicted in Fig. 2. As shown, for all the cases, the amplitude response to $\mathrm{X}$-rays is found to be linear in the regions below and above the $\mathrm{K}$-edges, presenting a discontinuity at the K-edges.

To determine the discontinuity, each straight line fit was extrapolated to the respective K-edge region. An abrupt decrease in the channel number was found in all cases. These discontinuities were converted into the corresponding energy using the average energy per channel established for each run. The measured discontinuities, based upon this method, were determined to be $0.5 \pm 0.1,1.7 \pm 0.3$ and $0.8 \pm 0.2 \mathrm{keV}$ for the YAP, $\mathrm{NaI}(\mathrm{Tl})$ and $\mathrm{BaF}_{2}$ scintillators, respectively. These discontinuities result in a region within $0.5 \pm 0.1,1.6 \pm 0.3$ and $0.9 \pm 0.2 \mathrm{keV}$ where the X-ray energy cannot be obtained unambiguously.

We note that, for the $\mathrm{NaI}(\mathrm{Tl})$ case, the La-Kpeak centroid lies between the two linear fittings. This is due to the energies of the $\mathrm{K}_{\alpha 1}$ and $\mathrm{K}_{\alpha 2}$, which are above and below the I-K-edge, respectively. The discontinuities obtained for the ionic crystals are noticeably higher than those obtained for gas detectors. In particular, the results obtained for the $\mathrm{NaI}$ and $\mathrm{BaF}_{2}$ are more than four times higher than those obtained for Xe [1]. The higher non-linearity effect observed for the amplitude response of $\mathrm{NaI}(\mathrm{Tl})$ may be attributed to the larger light yield non-proportionality in the electron response of this crystal. While for $\mathrm{NaI}(\mathrm{Tl})$ the scintillation yield for electrons may present variations as large as $10 \%[9,11,12,14,17]$, YAP scintillators present an uniform trend [14].

Since the centroid channel number (or amplitude), $A$, is proportional to the average number of photons, $N$, produced by X-rays with energy $E_{x}$, the scintillation yield is proportional to the $A / E_{x}$ ratio. In Fig. 3, the typical behaviour obtained for the $A / E_{x}$ ratio is depicted as a function of the $\mathrm{X}$-ray energy, for the regions around the $\mathrm{K}$ absorption edges of Y, I and Ba. The error-bars were estimated taking into account the fluctuations in the relative values of the $A / E_{x}$ ratios obtained for the different runs. As seen, an abrupt variation in the scintillation yield is observed for all the cases at the respective K-edge, characteristic 


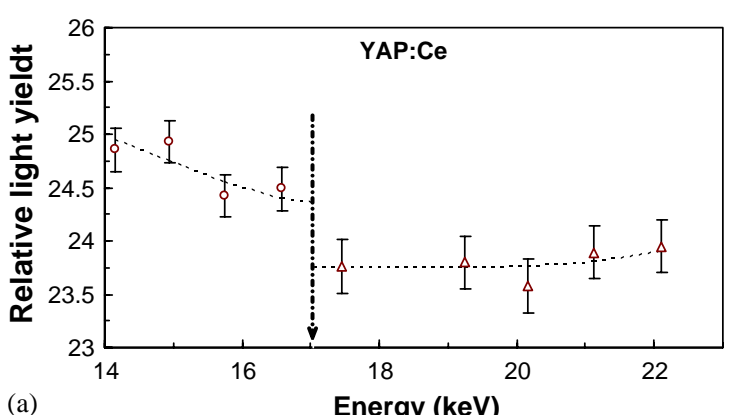

(a)
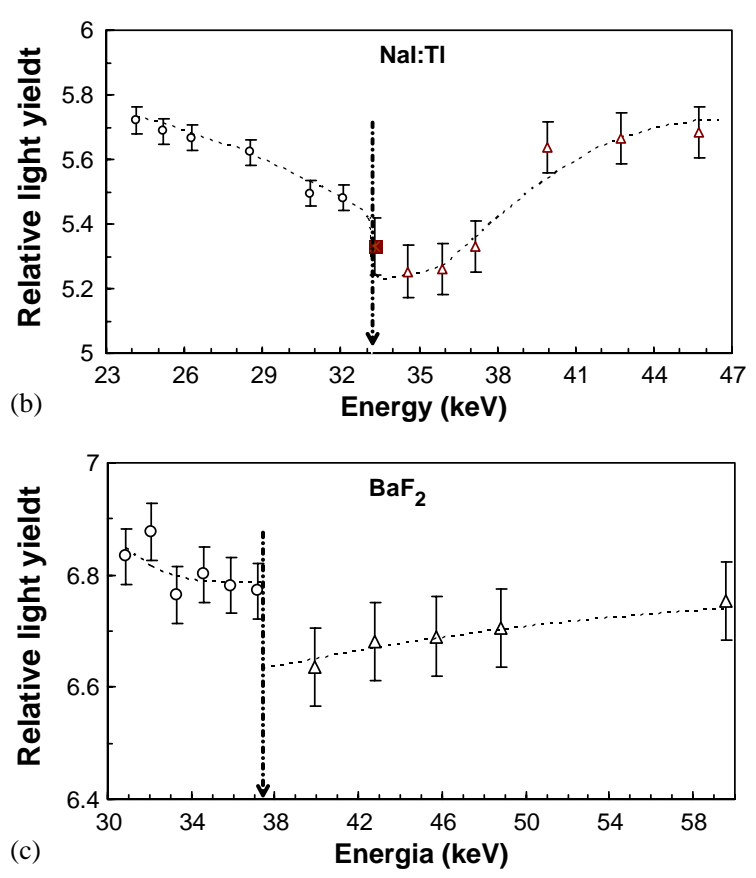

Fig. 3. Centroid channel-to- X-ray energy ratio, $\mathrm{A} / E_{x},(\bigcirc, \Delta)$ as a function of the X-ray energy, $E_{x}$ : (a) for $\mathrm{YAP},(\mathrm{b}) \mathrm{NaI}(\mathrm{Tl})$, (c) $\mathrm{BaF}_{2}$ scintillators, below $(O)$ and above $(\Delta)$ the respective K-absorption edges.

of a discontinuity in the detector response. Relative variations of $3 \%, 4 \%$ and $2 \%$ were measured for the YAP, $\mathrm{NaI}(\mathrm{Tl})$ and $\mathrm{BaF}_{2}$ scintillators, which are significantly higher than the $0.5 \%$ relative variation obtained for the $w$-value of $\mathrm{Xe}$ gas detectors.

The relative behaviour obtained for the $\mathrm{NaI}(\mathrm{Tl})$ scintillation yield is in good agreement with that presented in Ref. [12]. The results obtained for YAP and $\mathrm{BaF}_{2}$ show a nearly constant trend above and below the K-edge, which is in agreement with the behaviour depicted in Refs. [9,13]. The results obtained for YAP and $\mathrm{BaF}_{2}$ scintillators are consistent with a significantly more proportional response of these crystals to electrons. While for YAPs the literature confirms this trend [14], for $\mathrm{BaF}_{2}$ there is no data available in the literature, to the best of our knowledge.

\section{Conclusions}

We investigated the response of YAP, $\mathrm{NaI}(\mathrm{Tl})$ and $\mathrm{BaF}_{2}$ scintillators to $\mathrm{X}$-rays with energies around the $\mathrm{Y}$, I, and $\mathrm{Ba}$ K-absorption edges, respectively. The experimental results reveal a linear amplitude response of the detectors, except for the region around the K-absorption edges. An abrupt decrease of about 3\%,5\% and 2\% were observed in the detector amplitude at the K-edges, for the YAP, the $\mathrm{NaI}(\mathrm{Tl})$ and the $\mathrm{BaF}_{2}$ scintillator. These discontinuities result in a region within $0.5 \pm 0.1,1.6 \pm 0.3$ and $0.9 \pm 0.2 \mathrm{keV}$ where the X-ray energy cannot be obtained unambiguously. The scintillation yield was found to present an abrupt decrease of $3 \%, 4 \%$ and $2 \%$, respectively, at the K-edges. The measured non-linearity effects are more significant than those obtained for gaseous detectors. Additionally, the larger dependence in the electron response of $\mathrm{NaI}(\mathrm{Tl})$ results in higher measured amplitude discontinuity. The results obtained for YAP and $\mathrm{BaF}_{2}$ scintillators are consistent with a more proportional response of these crystals to electrons.

\section{Acknowledgements}

This work has been carried out in the Instrumentation Centre (unit 217/94) of the Physics Department, University of Coimbra. Support is acknowledged to Fundação para a Ciência e a Tecnologia (FCT) through project POCTI/FIS/ $1920 / 95$. J.F.C.A. Veloso acknowledges funding from FCT. We thank Prof. C.A.N. Conde (GIAN, University of Coimbra) for useful suggestions and discussions. 


\section{References}

[1] T.H.V.T. Dias, J.M.F. Dos Santos, P.J.B.M. Rachinhas, F.P. Santos, C.A.N. Conde, A.D. Stauffer, J. Appl. Phys. 82 (1997) 2742.

[2] K. Torii, H. Tsunemi, E. Miyata, K. Hayashida, Nucl. Instr. and Meth. A 361 (1995) 364.

[3] A. Owens, G.W. Fraser, A.F. Abbey, A. Holland, K. McCarthy, A. Keay, A. Wells, Nucl. Instr. and Meth. A 382 (1996) 503.

[4] J.M.F. Dos Santos, C.M.B. Monteiro, Appl. Rad. Isot. 55 (2001) 331.

[5] E.D.C. Freitas, João Cardoso, L.M.P.Fernandes, J.A.M. Lopes, J.F.C.A. Veloso, J.M.F. dos Santos, Energy linearity response of CZT detectors to X-rays with energy in the region of $\mathrm{Zn}, \mathrm{Cd}$ and $\mathrm{Te} \mathrm{K}$-absorptions edges: experimental results, submitted for publication to Nucl. Instr. and Meth. A. (2003) in press.

[6] C.M.B. Monteiro, P.C.P.S. Simões, J.F.C.A. Veloso, J.M.F. dos Santos, C.A.N. Conde, Nucl. Instr. and Meth. A 505 (2003) 233.

[7] G.W. Fraser, A.F. Abbey, A. Holland, K. McCarthy, A. Owens, A. Wells, Nucl. Instr. and Meth. A 350 (1994) 368.
[8] F.P. Santos, T.V.H.T. Dias, A.D. Stauffer, C.A.N. Conde, Nucl. Instr. and Meth. A 307 (1991) 347.

[9] P. Dorenbos, J.T.M. de Haas, C.W.E. van Eijk, IEEE Trans. Nucl. Sci. 42 (1995) 2190.

[10] H. Leutz, C. D’Ambrosio, IEEE Trans. Nucl. Sci. NS-44 (1997) 190

[11] J.D. Valentine, B.D. Rooney, P. Dorenbos, IEEE Trans. Nucl. Sci. NS-45 (1998) 1750.

[12] L.R. Wayne, W.A. Heindl, P.L. Hink, R.E. Rothschild, Nucl. Instr. and Meth. A 411 (1998) 351.

[13] P. Dorenbos, J.T.M. De Haas, C.W.E. Van Eijk, Radiat. Meas. 24 (1995) 355.

[14] W. Mengesha, T.D. Taulbee, B.D. Rooney, J.D. Valentine, IEEE Trans Nucl. Sci. NS-45 (1998) 456.

[15] E.V.D. van Loef, W. Mengesha, J.D. Valentine, P. Dorenbos, C.W.E. van Eijk, IEEE Trans. Nucl. Sci. NS50 (2003) 155.

[16] P.R. Bevington, Data Reduction and Error Analysis for the Physical Sciences, McGraw-Hill, New York, 1969 , pp. 208-214.

[17] B.D. Rooney, J.D. Valentine, IEEE Trans. Nucl. Sci. NS44 (1996) 509. 